\title{
Sequence analysis of Indian SARS-CoV-2 isolates shows a stronger interaction of mutated receptor binding domain with $\mathrm{ACE} 2$ receptor
}

Pujarini Dash ${ }^{1}$, Jyotirmayee Turuk*1, Santosh Ku. Behera ${ }^{1}$, Subrata Ku. Palo ${ }^{1}$, Sunil K. Raghav $^{*}{ }^{2}$, Arup Ghosh ${ }^{2}$, Jyotsnamayee Sabat ${ }^{1}$, Sonalika Rath ${ }^{1}$, Subhra Subhadra ${ }^{1}$, Debadutta Bhattacharya ${ }^{1}$, Srikant Kanungo ${ }^{1}$, Jayasingh Kshatri ${ }^{1}$, Bijaya kumar Mishra ${ }^{1}$, Saroj Dash ${ }^{1}$, Namita Mahapatra ${ }^{1}$, Ajay Parida ${ }^{2}$, Sanghamitra Pati ${ }^{1}$

${ }^{1}$ Regional Medical Research Centre, Indian Council of Medical Research, Bhubaneswar, Odisha, India

${ }^{2}$ Institute of Life Sciences, Nalco square, Chandrasekharpur, Bhubaneswar, and Odisha, India

*Author for correspondence: Dr. Jyotirmayee Turuk, Dr. Sunil K. Raghav

ICMR-Regional Medical Research Centre,

Chandrasekharpur, Bhubaneswar - 751023,

Odisha, India. 


\begin{abstract}
SARS-CoV-2 is a RNA Coronavirus responsible for the pandemic of the Severe Acute Respiratory Syndrome (COVID-19). It has affected the whole world including Odisha, a state in eastern India. Many people migrated in the state from different countries as well as states during this SARS-CoV-2 pandemic. As per the protocol laid by ICMR and Health \& Family welfare of India, all the suspected cases were tested for SARS-CoV-2 infection. The aim of this study was to analyze the RNA binding domain (RBD) sequence of spike protein from the isolates collected from the throat swab samples of COVID-19 positive cases and further to assess the RBD affinity with ACE2 of different species including human.

Whole genome sequencing for 35 clinical SARS-CoV-2 isolates from COVID-19 positive patients was performed using ARTIC amplicon based sequencing. Sequence analysis and phylogenetic analysis was carried out for the Spike and RBD region of all isolates. The interaction between the RBD and ACE2 receptor of five different species was also analysed.

Except three isolates, spike region of 32 isolates showed one/multiple alterations in nucleotide bases in comparison to the Wuhan reference strain. One of the identified mutation at 1204 (Ref A, RMRC 22 C) in the RBD of spike protein was identified which depicted a stronger binding affinity with human ACE2 receptor compared to the wild type RBD. Furthermore, RBDs of all the Indian isolates are capable of binding to ACE2 of human, bat, hamster and pangolin.
\end{abstract}

As mutated RBD showed stronger interaction with human ACE2, it could potentially result in higher infectivity. The study shows that RBDs of all the studied isolates have binding affinity for all the five species, which suggests that the virus can infect a wide variety of animals which could also act as natural reservoir for SARS-CoV-2.

Key words: SARS-COV-2, Indian isolates, Spike, RBD, ACE2 


\section{Introduction}

Corona viruses have been studied for more than fifty years and are known to infect multiple animal species including human. Though their pathogenesis and mechanism of replication has already been well described due to previous outbreaks (SARS-CoV in China, 2003 and MERS-CoV in Saudi Arabia, 2012), the current pandemic has propelled the whole world to research and investigate deeper into the pathogenesis of this virus. Coronaviruses belong to a diverse virus family which consists of four genera: Alpha, Beta, Gamma and Delta corona viruses ${ }^{1}$. According to the nucleic acid sequence similarity, SARS-CoV-2 or COVID-19 is a beta corona virus. The spike glycoprotein of corona viruses facilitates its entry into their host cell and also gives the virus a crown like structure on its surface. Binding of pathogenic particles of COVID-19 with host cell receptors remains the crucial step for initiation of infection. The key factor lies in receptor recognition and establishment of infection in cells or tissues. Besides, the ability of the virus to bind to the specific receptor of other host species is also an essential requirement for transmission across different species ${ }^{2}$. It is known that SARS-CoV interacts with human angiotensin converting enzyme 2 (ACE2) for their entry and after COVID-19 outbreak, researchers found that SARS-CoV-2 also interacts with ACE2 for the viral entry into host cells ${ }^{3,4}$. SARS-CoV-2 spike (S) protein gets cleaved by host proteases into $\mathrm{S} 1$ and $\mathrm{S} 2$ domain which mediates receptor recognition and membrane fusion respectively ${ }^{5}$. Wang et al. (2020) reported that, the S1 domain of S protein of SARS-CoV-2 contains a region called receptor binding domain (RBD) which makes a complex with human ACE2 and facilitates viral entry ${ }^{6}$. However, emerging mutations in SARS-CoV-2 genome might alter the process of infection transmission, replication and potential of viral attachment with ACE2. According to epidemiological data, COVID-19 has an origin from bats in Wuhan, China and then spread to other parts after its zoonotic transmission via the Malayan Pangolins ${ }^{7,8}$. In a country like India having a diversified geographical distribution, it is important to understand the pathogenesis of different strains of SARS-CoV-2 isolated from different parts of the country. As interaction with ACE2 is the main pathway of entry of this virus into its host, knowledge on the RBD binding affinity of different Indian SARS-CoV-2 isolates with the ACE2 of their natural reservoirs including human is highly essential. However, this has remained a grey area with a little information available. In the current study, we have analysed the RBD sequences of spike protein from different isolates of 
SARS-CoV-2 from COVID-19 patients of Odisha. Further we analysed the binding affinity of RBDs with the ACE2 of different probable natural hosts of corona virus: bat, pangolin and hamster including human. The detected mutation in the RBD region of one isolate shows stronger binding affinity with human ACE2 than the wild type RBD, providing important information regarding its virulence as well as drug targeting.

\section{Material \& Methods}

\section{Sequencing of different Indian isolates of SARS-CoV-2 from throat swab samples}

The current study was a part of whole genome sequencing study carried under the Odisha Study group constituting different Government organisations jointly by Regional Medical Research Centre (RMRC), Bhubaneswar and Institute of Life Sciences, Bhubaneswar. As ICMR-RMRC, Bhubaneswar is a govt. authorised testing laboratory for COVID-19 testing, we received throat swab samples of suspected cases from different hospitals of Odisha. For COVID-19 diagnosis, viral RNA isolation was carried out from all samples using QIAmp Viral RNA Mini Kit followed by qPCR (Taqpath ${ }^{\mathrm{TM}}$ 1-step Master Mix, ThermoFisher Scientific). The libraries were prepared for WGS using ACTIC amplicon based sequencing kits from Qiagen as per manufacturer recommended protocol. Out of all positive samples, whole genome sequencing of 35 isolates from different COVID-19 patients with different travel history, was carried out using Illumina platform. The detailed information and method have been described by Raghav et al., $2020^{9}$

\section{Sequence alignment of RMRC spike genes with reference sequence}

For sequence alignment, full length genome sequence of SARS-CoV-2 isolate of Wuhan-Hu1 (Accession no.NC_045512) was downloaded from NCBI database and used as reference sequence for all further analysis. Alignment of all 35 RMRC spike nucleotide sequences with the reference genome was carried out using BLASTN (align two/more sequences).

\section{Identification and phylogenetic analysis of RBD of RMRC SARS-CoV-2 isolates}

From the data available for reference strain, we retrieved the information of coding region of spike RBD domain sequence and all RMRC spike sequences were aligned with the reference sequence to identify the respective RBD mutations using BLASTN. Multiple sequence alignment of all 35 RBDs (amino acid sequences) was carried out using Clustal X tool. Mutations specific to RMRC isolates were identified by comparing the RBD coding regions 
with the reference strain. A phylogenetic tree was generated using the MEGA software version 6 with 1000 bootstrap replications as instructed in MEGA software.

\section{Sequence and 3D structure analysis of ACE2 receptor}

From the phylogenetic tree analysis, four RMRC RBD sequences were selected from four random clusters for further investigation of their interactions with ACE2 receptor of probable natural hosts of SARS-CoV-2. Hamsters were reported to be the best suitable animal model to carry out SARS-CoV-2 related experiments; therefore it was essential to understand the interaction of its ACE2 receptor with isolated Indian SARS-CoV-2 RBDs ${ }^{10}$. For the interaction study, hamster (Mesocricetus auratus) (UniprotKB-C7ECV1, 785 amino acids), pangolin (Manis javanica) (NCBIXP_017505752, 805 amino acids), Chinese bat (Rhinolophus sinicus) (Uniprot E2DHI7, 805 amino acids), Indian bat (Cynopterus sphinx) (Uniprot QZF77831, 807 amino acids) (probable natural hosts for SARS-CoV-2) and human (Homo Sapiens) ACE2 (UniprotKB Q9BYF1, 805 amino acids) sequences were included. Before inception of structure prediction, the ACE2 sequences from pangolin, hamster, Chinese and Indian bat were aligned with the human counterpart to identify the percentage of similarity and dissimilarity between these sequences.

The experimental 3D structure of human ACE2 was retrieved from RCSB PDB (PDB ID-6M0J) with a resolution of $2.45 \AA$ positioning from 19-615 amino acids. Protein Data Bank (PDB) did not provide any experimental structure of ACE2 receptors of pangolin, hamster, Chinese and Indian bat, which has prompted us to predict their three dimensional (3D) structure through homology modelling using Modeller v 9.19 tool followed by structure validation. Suitable templates were identified for 3D model building of ACE2 using BLASTp ${ }^{11}$ search against PDB (Protein Data Bank) database. The templates with PDB ID: 1R42, 6CS2, 6LZG, 3SCI, and 2AJF, were found to be the better homologs for target-template alignment and modelled 3D structure prediction. Based on the optimized target-template alignment, Modeller $\mathrm{v} 9.19^{12}$ facilitated in model developments, the models with lowest Discrete Optimized Protein Energy (DOPE) score were retained for further structural refinement. Side chain optimization was performed using WHATIF $^{13}$ and GalaxyRefine ${ }^{14}$ tool. The optimized models of ACE2 were finalized based on its overall quality and stereo-chemical geometry and energy. The geometry of the predicted model was evaluated using PROCHECK ${ }^{15}$ and Ramachandran analysis ${ }^{16}$. ERRAT $^{17}$ programme was used to calculate the accuracy of the nonbonded atoms for the predicted model. Verify $3 \mathrm{D}^{18}$ tool was used to evaluate the compatibility 
of the 3D model with its own amino acid sequence by assigning a structural class based on its location, environment and comparing the results to good quality structures. The structure was uploaded in Qualitative Model Energy Analysis (QMEAN) server (bencrept), for resolving the model quality. The energy potential of the predicted model was calculated using ProSA-web server $^{19}$.

\section{In silico translation of RBD sequences and their interaction with ACE2 receptor of different natural reservoirs}

Four nucleotide sequences of RMRC RBDs were selected and translated to protein sequence using EMBOSS Transeq tool of European Bioinformatics Institute (EBI). The 3-D structure of reference human RBD (Uniprot ID: PODTC2, 333-526 amino acids of the spike protein, PDB ID-6M0J) was considered as wild type. The experimental structure of the wild RBD was mutated at the position I402L using Discovery Studio visualizer (4.1) for attaining a mutant RMRC22 RBD as required for further computational analysis. Finally, study of protein-protein interaction (PPI) between wild type/mutant RBDs and ACE2 receptors form different organisms was carried out using online HawkDock server which is a powerful tool to predict the binding structures and identify the key residues of PPIs ${ }^{20}$.

\section{Results}

\section{Sequence information and analysis of spike gene of Indian SARS-CoV-2 isolates}

All the SARS Co-V-2 isolates included in the study had a travel history from either outside the country or from other states of India. According to the travel history, out of 35 isolates, one had a foreign travel history, 11 were Nizamuddin (cluster detected from New Delhi, India during April, 2020) returned and rest 23 migrated from Surat, Gujarat, India. The detail of the demographic and clinical status of the patients included in the study has been described in one of our unpublished reports (Turuk et al). We did not record any death case among the patients whose samples were included in the current study.

. In the current study, the spike region was identified at the position from 21563-25384 of the whole genome and consists of 3822 nucleotides. The BLAST alignment analysis of RMRC spike nucleotide sequences alignment analysis showed that, three spike sequences (RMRC 
$104,157,158)$ were $100 \%$ identical to the Wuhan reference spike whereas all other RMRC spikes shared $99 \%$ identity with one or multiple altered bases at different positions (Table 1).

\section{Sequence identification, alignment and phylogenetic analysis of RBD}

The alignment of the spike sequences of RMRC isolates with the reference strain RBD, revealed the RBD region of Indian SARS-CoV-2 isolates sitting at 996-1578 bp region of the spike gene consisting of total 582 bases. The protein sequence of the RBD spans from 333 (T, Threonine) - 526 (G, Glycine) amino acids of the spike protein ${ }^{21}$. Interestingly RBD sequence alignment results showed that, RMRC 22 has $99 \%$ sequence identity with the reference RBD and harbours a mutation at 1204 (Nucleotide: Ref A, RMRC 22 C; Protein: Ref I, RMRC 22 L) (Fig.1A). Except for RMRC 22, other three shared 100\% identity with the reference RBD (Uniprot ID: PODTC2, 333-526 amino acids of the spike protein). As only RMRC 22 had a mutation, it was named as mutant isolate, whereas all other isolates were considered as wild type.

The phylogenetic analysis was carried out using RBD sequences of all 35 RMRC isolates which showed that they formed four different clusters. RMRC 22 having a mutation belonged to first cluster, RMRC 171 RBD formed second cluster, four RBDs (RMRC 2, 5, 6 and 7) grouped in the third cluster and all other RBDs were in cluster four, which describes their phylogenetic distribution (Fig.1B).

\section{Protein structure analysis and interaction of RBD with ACE2 receptor}

The sequence alignment analysis showed that ACE2 of pangolin, hamster, Chinese and Indian bat shared $85 \%, 84 \%, 80 \%$ and $78 \%$ identity with human ACE2 respectively (Suppl. Fig.1). The quality of modelled structures of ACE2 was validated using several computational methods (pangolin Fig.2A; hamster Fig.3A; Chinese bat Fig.4A; Indian bat Fig. 5A; human Fig.6A). In Pangolin, out of 805 amino acid residues, the Ramachandran analysis illustrated $637(88.0 \%)$ residues in most favoured regions, $66(9.1 \%)$ in additional allowed regions, 14 $(1.9 \%)$ in generously allowed regions and 7 (1.0\%) in disallowed regions (Fig. 2B). In Hamster, out of 785 amino acid residues, the Ramachandran analysis illustrated 613 (87.2\%) residues in most favoured regions, $70(10.0 \%)$ in additional allowed regions, $12(1.7 \%)$ generously allowed regions and $8(1.1 \%)$ in disallowed regions (Fig.3B). In Chinese bat, out of 805 amino acid residues, the Ramachandran analysis illustrated 634 (88.8\%) residues in most 
favoured regions, $66(9.2 \%)$ in additional allowed regions, 12 (1.7\%) generously allowed regions and $2(0.3 \%)$ in disallowed regions (Fig.4B). In Indian bat, out of 807 amino acid residues, the Ramachandran analysis illustrated $630(86.8 \% 88.8 \%)$ residues in most favoured regions, $73(10.1 \%)$ in additional allowed regions, $15(2.1 \%)$ generously allowed regions and 8 $(1.1 \%)$ in disallowed regions (Fig.5B). Using the Qualitative Model Energy Analysis (QMEAN) server, the model quality was determined. The overall quality of model was good as indicated by its QMEAN Z-score and QMEAN4 global score. Low quality models are expected to have a negative QMEAN Z-score. The QMEAN4 ranges from 0 to 1 and a higher value indicates good qualitymodel ${ }^{22}$. Additionally, the overall quality of the model was evaluated using Protein Structure Analysis (ProSA) tool which provides a quality score (Zscore) as compared to all known protein structure from x-ray crystallography as well structural NMR. The obtained Z-score value was -6.33 (Pangolin), -4.76 (Hamster), -6.24 (Chinese bat) and -6.73 (Indian bat); which indicates the high quality of the models compared to known protein structures (Fig. 2B-5B).

As the 3D structure of human RBD was already available in the database (PDB ID-6M0J), we inserted the observed mutation of RMRC 22 isolate (Protein: Ref I, RMRC 22 L) and predicted the structure of mutated RBD (Fig. 6B). As the specific binding of viral RBD with host ACE2 receptor determines the establishment of infection, we analysed the interaction of Indian SARS-CoV-2 RBDs (both mutant and wild type) with the ACE2 receptor of human as well as other species which are reported to be natural reservoirs for this virus. The interaction analysis showed that, the mutated RBD of RMRC 22 isolate has stronger interaction with human ACE2 (Fig. 6D) as compared to the wild type RBD (Fig. 6C). The interaction between mutated RBDhuman ACE2 has a binding energy of $-65.95 \mathrm{Kcal} / \mathrm{Mol}$ with six hydrogen bonds whereas wild type RBD-human ACE2 interaction has $-63.09 \mathrm{Kcal} / \mathrm{Mol}$ binding energy with four hydrogen bonds. For ACE2 receptor of all other species (Pangolin: Fig. 2C \& 2D; hamster: Fig.3C \& 3D; Chinese bat: Fig.4C \& 4D; Indian bat: Fig.5C \& 5D), wild type RBD seems to have stronger binding affinity with no difference in number of hydrogen bonds (except hamster, wild type RBD-ACE2:2 and mutant RBD-ACE2: 3 hydrogen bonds). The details of the interaction analysis with $\mathrm{H}$-bond forming residues and average distance of $\mathrm{H}$-bonds has been described in Table-2. However, if we compare the interaction of mutated and wild type RBD with ACE2 receptor of all species, the interaction between mutated RBD and human ACE2 is the strongest one with highest binding energy and highest number of hydrogen bonds. 


\section{Discussion}

The complex trajectory of the recent COVID-19 pandemic in India poses greater risk towards control and containment of the infection. It is high time to understand its mobility pattern in the country and the viral genetic properties favouring its virulence. Though during early phase of pandemic, Odisha, had comparatively very few positive cases as well as small number of deaths, gradually, the virus has become rapidly infectious making the clinical scenario worsen. Many people from Odisha were working outside the state and during this pandemic, they returned to their home state due to many reasons. The samples included in our study were mainly collected from suspected cases who had a travel history from different states with one foreign travel record.

As spike protein of SARS-CoV-2 mediates viral entry in to host and houses RBD, which binds to ACE2 receptor of host cell, understanding the spike-RBD distribution in the genome of Indian isolates is crucial for therapeutic design. SARS-CoV-2 spike protein is reported to have a stronger binding affinity for ACE2 than SARS-CoV and higher affinity means low number of virus is required to infect the cell, which may explain the high transmission of SARS-CoV- $2^{23}$. In the current study, spike region of 32 isolates showed altered nucleotide bases at multiple positions as compared to the Wuhan reference strain suggesting mutations in these Indian isolates during the spread. For SARS-CoV-2, specific RBD-ACE2 binding ensures infection as well as serves as a potential target for developing treatment strategies for this infection ${ }^{23}$. According to Premkumar et al. 2020, the RBD of SARS-CoV-2 is an immunodominant and a potential target of antibodies in COVID-19 patients ${ }^{24}$. The mutation found in RMRC 22 isolate might play a role in altering the antigenicity or binding affinity of the respective RBD. Due to the rapid spreading and evolution, SARS-CoV-2 RBD is known to acquire several mutations leading to increased binding affinity to human ACE2 receptor ${ }^{25}$. In France, multiple mutations were identified in RBD of SARS-CoV-2 contributing to higher receptor binding capacity, which might be responsible for increased virus spread and infectivity $^{26}$. On the other hand, a mutation in $\mathrm{S}$ protein has been found to be associated with decrease in receptor binding affinity ${ }^{27,28}$. In the current study, mutation in the RBD region of Indian isolates did not seem to affect its interaction with ACE2 receptor of other species prominently except that of human. Surprisingly, the patient, from whom, the RMRC 22 isolate was obtained, had a travel history of returning from Nizamuddin (cluster detected from New Delhi, India during April, 2020) recently before he tested positive for SARS-CoV- 
2. However, no mutation was observed in the isolates obtained from his other family members (his father and two brothers) who were also COVID-19 positive. It appears that, emergence and role of a mutation in any region of SARS-CoV-2 genome depends upon multiple factors including the geographical distribution, rate of spreading, alteration in the virulence of the virus and immune response of the host. The interaction analysis of mutated and wild type RBDs with ACE2 receptor indicated that bats and pangolins could be suitable natural reservoirs for Indian isolates of this virus and this finding falls parallel with other earlier reports ${ }^{29,30}$. As hamster has been reported as a suitable animal model to study SARSCoV-2 pathogenesis, the interaction of RBD of the Indian isolates included in the current study makes the earlier report more relevant ${ }^{10}$. Though the susceptibility of infection and death rate could be affected by several factors, mutation in the virus genome and its ability to adapt to new environment could be crucial.

Being an important determinant in SARS-CoV-2 infection, RBD-ACE2 interaction has already become the potential target for developing treatment therapy against this deadly pathogen. The current study provides important information regarding the structural basis of spike and RBD regions of few Indian SARS-CoV-2 isolates which gives an idea about their evolution and spreading. The mutation observed in the RBD region of one of the isolates sheds light on drug targeted therapy for different strains of virus. Further studies with larger number of isolates of a more wide origin would be helpful to understand this mutation pattern in RBD of Indian isolates.

Acknowledgment: We acknowledge the contribution of all the VRDL staff (Laboratory technicians, Multi tasking workers) whose relentless effort in COVID testing has given the strength to further plan up new experiments and studies. We also acknowledge the support of Dr. L.M. Ho for his contribution. We acknowledge Dr. Ira Praharaj and Dr. Sidharth Giri for their expert advice and scientific inputs.

\section{Financial support \& sponsorship: None}

\section{Conflicts of Interest: None.}




\section{References}

1. Fehr AR, Perlman S. Coronaviruses: an overview of their replication and pathogenesis. Methods Mol Biol 2015; 1282 : 1-23.

2. Lu G, Wang Q, Gao GF. Bat-to-human: spike features determining 'host jump' of coronaviruses SARS-CoV, MERS-CoV, and beyond. Trends Microbiol 2015; 23 : $468-78$.

3. Li W, Moore MJ, Vasilieva N, Sui J, Wong SK, Berne MA, et al. Angiotensinconverting enzyme 2 is a functional receptor for the SARS coronavirus. Nature 2003; $426: 450-54$.

4. Zhou P, Yang XL, Wang XG, Hu B, Zhang L, Zhang W, et al. A pneumonia outbreak associated with a new coronavirus of probable bat origin. Nature 2020; 579 : 270-73.

5. Lai MMC, Perlman S, Anderson LJ. (2007). Coronaviridae. In Fields Virology, D.M. Knipe and P.M. Howley, eds. (Lippincott Williams \& Wilkins), pp. 1305-1335.

6. Wang Q, Zhang Y, Wu L, Niu S, Song C, Zhang Z, et al. Structural and Functional Basis of SARS-CoV-2 Entry by Using Human ACE2. Cell 2020; 181: 894-904.

7. Chinazzi M, Davis JT, Ajelli M, Gioannini C, Litvinova M, Stefano M, et al. The effect of travel restrictions on the spread of the 2019 novel coronavirus (COVID-19) outbreak. Science 2020; $368: 395-400$.

8. Zhang T, Wu Q, Zhang Z. Probable pangolin origin of SARS-CoV-2 associated with the COVID-19 outbreak. Curr Biol 2020; 30:1346-51.

9. Raghav S, Ghosh A, Turuk J, Kumar S, Jha A, Madhulika S, et al. SARS-CoV2 genome analysis of Indian isolates and molecular modelling of D614G mutated spike protein with TMPRSS2 depicted its enhanced interaction and virus infectivity. bioRxiv preprint doi: https://doi.org/10.1101/2020.07.23.217430bn. 
10. Imai M, Imai-Kiyoko M, Iwatsuki-Horimoto K, Hatta M, Loeber S, Halfmann PJ, et al. Syrian hamsters as a small animal model for SARS-cov2 infection and countermeasure development. PNAS 2020; 117(28) : 16587-95.

11. Altschul SF, Gish W, Miller W, Myers EW, Lipman DJ. 1990. Basic Local Alignment Search Tool. J Mol Biol 1990; 215 : 403-10.

12. Webb B, Sali A. Comparative Protein Structure Modeling Using MODELLER. Curr Protoc Bioinforma 2017; 54 : 1-55.

13. Hekkelman ML, te Beek TH, Pettifer S, Thorne D, Attwood TK, Vriend G. WIWS: A protein structure bioinformatics web service collection. Nucleic Acids Res 2010; 38 : 719-23.

14. Heo L, Park H, Seok C. 2013. GalaxyRefine: Protein structure refinement driven by side-chain repacking. Nucleic Acid Res 2013; 41 : 384-8.

15. Laskowski RA, MacArthur MW, Moss DS, Thornton JM. 1993. PROCHECK: a program to check the stereochemical quality of protein structures. J Appl Crystallogr $1993 ; 26: 283-91$.

16. Pontius J, Richelle J, Wodak SJ. Deviations from Standard Atomic Volumes as a Quality Measure for Protein Crystal Structures. J Mol Biol 1996; 264 : 121-36.

17. Colovos C, Yeates T. 1993. Verification of protein structures: patterns of nonbonded atomic interactions. Protein Sci 1993; 2 : 1511-9.

18. Bowie JU, Lüthy R, Eisenberg D. 1991. A method to identify protein sequences that fold into a known three-dimensional stucture. Science 1991; 253 : 164-70.

19. Wiederstein M, Sippl MJ. 2007. ProSA-web: Interactive web service for the recognition of errors in three-dimensional structures of proteins. Nucleic Acids Res 2007; 35: 407-10.

20. Weng G, Wang E, Wang Z, Liu H, Zhu F, Li D, et al. HawkDock: a web server to predict and analyze the protein-protein complex based on computational docking and MM/GBSA. Nucleic Acids Res 2019; 47 : 322-30.

21. Lan J, Ge J, Yu J, ShanS, Zhou H, Fan S, et al. Structure of the SARS-CoV-2 spike receptor-binding domain bound to the ACE2 receptor. Nature 2020; https://doi.org/10.1038/s41586-020-2180-5 (2020).

22. Benkert P, Tosatto SCE, Schomburg D. QMEAN: A comprehensive scoring function for model quality assessment. Proteins Struct Funct Genet 2008; 71 : 261-77. 
23. Chen Y, Guo Y, Pan Y, Zhao ZJ. Structure analysis of the receptor binding of 2019nCoV. Biochem Bioph Res Co 2020; 525 : 135-40.

24. Premkumar L, Segovia-Chumbez B, Jadi R, Martinez DR, Raut R, Markmann AJ, Cornaby C, et al. The receptor-binding domain of the viral spike protein is an immunodominant and highly specific target of antibodies in SARS-CoV-2 patients. Science Immunol 2020; 5(48) : eabc8413. DOI: 10.1126/sciimmunol.abc8413

25. Ortega JT, Serrano ML, Pujol FH, Rangel HR. Role of changes in sars-cov-2 spike protein in the interaction with the human ace 2 receptor: an in silico analysis. Excli Journal 2020; $19: 410-7$.

26. Ou J, Zhou Z, Dai R, Zhang J, Lan W, Zhao S, et al. Emergence of RBD mutations in circulating SARS-CoV-2 strains enhancing the structural stability and human ACE2 receptor affinity of the spike protein. BioRxiv 2020

27. Jia Y, Shen G, Zhang Y, Huang K-S, Ho H-Y, Hor WS, et al. Analysis of the mutation dynamics of SARS-CoV-2 reveals the spread history and emergence of RBD mutant with lower ACE2 binding affinity. bioRxiv 2020; doi.org/10.1101/2020.03.15.991844.

28. Saha P, Banerjee AK, Tripathi PP, Srivastava AK, Ray U. A virus that has gone viral: Amino acid mutation in S protein of Indian isolate of Coronavirus COVID-19 might impact receptor binding, and thus, infectivity. Biosci Rep 2020;40 : Doi: 10.1042/BSR20201312.

29. Zhou P, Yang XL, Wang XG, Hu B, Zhang L, Zhang W, et al. A pneumonia outbreak associated with a new coronavirus of probable bat origin. Nature 2020; $579: 270-3$.

30. Lam TT-Y, Shum MH-H, Zhu H-C, Tong Y-G, Ni X-B, Liao Y-S, et al. Identification of 2019-nCoV related coronaviruses in Malayan pangolins in southern China. Nature 2020; $583: 282-5$. 


\section{Figure legends}

Figure 1: (A) Multiple sequence alignment and (B) phylogenetic analysis of RBD sequences of RMRC isolates.

Figure 2: 3D structure analysis and interaction of ACE2 receptor of Manis javanica with wild and mutated RBD. (A) Predicted 3D structure of ACE2 receptor; (B) Ramachandran plot and Z-score; (C) ACE2 interaction with wild type RBD and (D) mutated RBD.

Figure 3: 3D structure analysis and interaction of ACE2 receptor of Mesocricetus auratus with wild and mutated RBD. (A) Predicted 3D structure of ACE2 receptor; (B) Ramachandran plot and Z-score; (C) ACE2 interaction with wild type RBD and (D) mutated RBD.

Figure 4: 3D structure analysis and interaction of ACE2 receptor of Rhinolophus sinicus with wild and mutated RBD. (A) Predicted 3D structure of ACE2 receptor; (B) Ramachandran plot and Z-score; (C) ACE2 interaction with wild type RBD and (D) mutated RBD.

Figure 5: 3D structure analysis and interaction of ACE2 receptor of Cynopterus sphinx with wild and mutated RBD. (A) Predicted 3D structure of ACE2 receptor; (B) Ramachandran plot and Z-score; (C) ACE2 interaction with wild type RBD and (D) mutated RBD.

Figure 6: 3D structure and interaction of ACE2 receptor of Homo Sapiens with wild and mutated RBD. (A) 3D structure of ACE2 receptor; (B) predicted structure of wild and mutated RBD of RMRC SARS-CoV-2 isolates; (C) H. sapiens ACE2 interaction with wild type RBD and (D) mutated RBD.

Supplementary figure: Sequence alignment of ACE2 receptors of Manis javanica, Mesocricetus auratus, Rhinolophus sinicus, Cynopterus sphinx and Homo Sapiens. 
File: C:CsersRMRCDesktop RBDprotein RMRC alignment fileMSA.ps Page 2 of 4
Date: Tue Jun 30 16:17:042020

1e11Quecy raec166 $599-1578$ 1ellojery Jaac167, $957-1571$

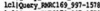

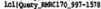
delipuery Joacin $997-1570$

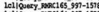

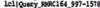
leliogery pac163 $597-1571$ 1el | Query RaAC162 957 -1571

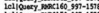
leliouery_raaciss, $957-157$ lel logery Bac154 $9 n 7-157$ 1eliouery aeac157 $5 n-159$

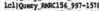
lellouery baec15s $997-157$

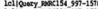
lel Q Query BaRC112 $997-1574$

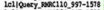
del Query yoacios $597-157$ deliguery yolcior $537-1578$

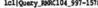

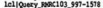

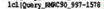
1el (Gutry nocets $597-1578$

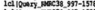

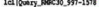

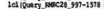
1el lowery NoC27 $597-1378$ lel lovery nioc2s $597-1570$ lel Duary nec24 $597-1570$

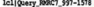
lell 0005y BabC6 $997-1571$ lell poery Jobes, $597-157 \%$ icligoery $95 a C 2$ 997-1578 led lovery $\sec 22$ 537-157

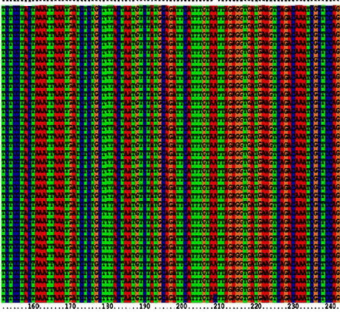

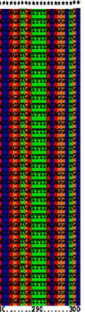

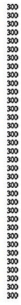




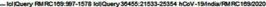

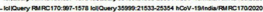

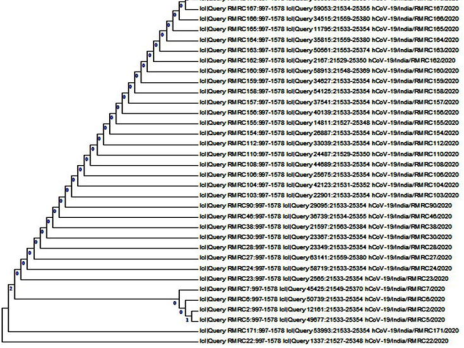


Figure 2

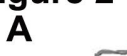

C

$$
\text { Manisavanica ACE2 }
$$

M. javanica ACE2-Wild RBD Complex

B

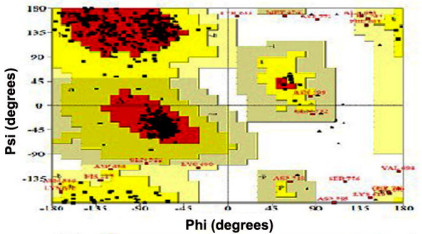

D

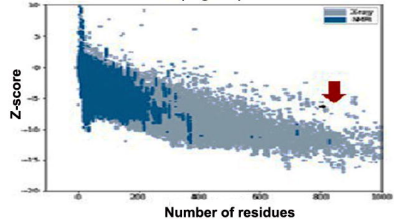

Mutated RBD

M. javanica ACE2-Mutated RBD Complex 
Figure 3

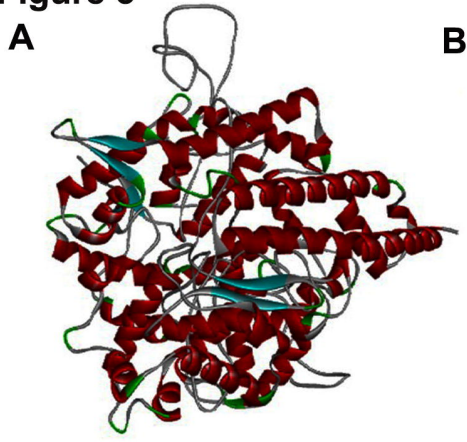

C

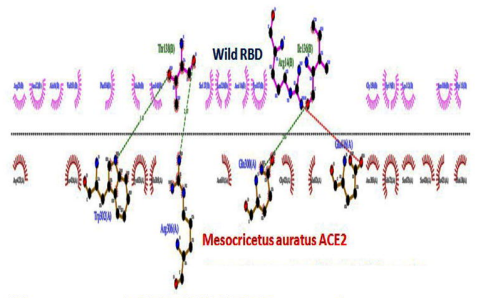

M. auratus ACE2-Wild RBD complex
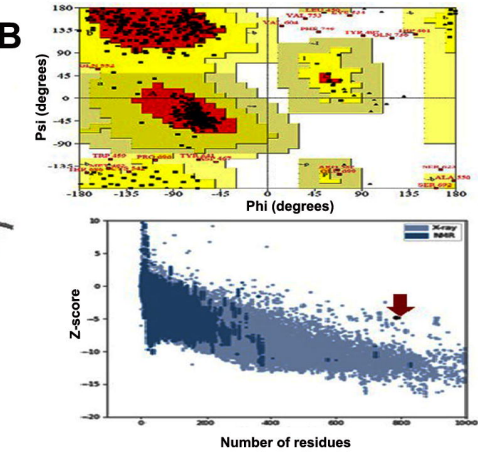

D

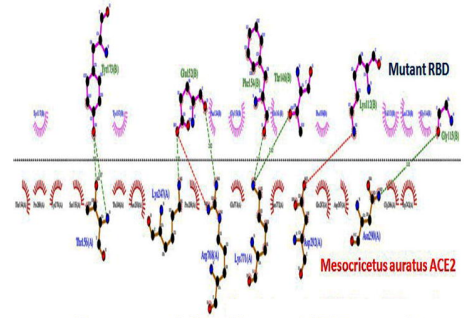

M. auratus ACE2-Mutated RBD complex 
Figure 5
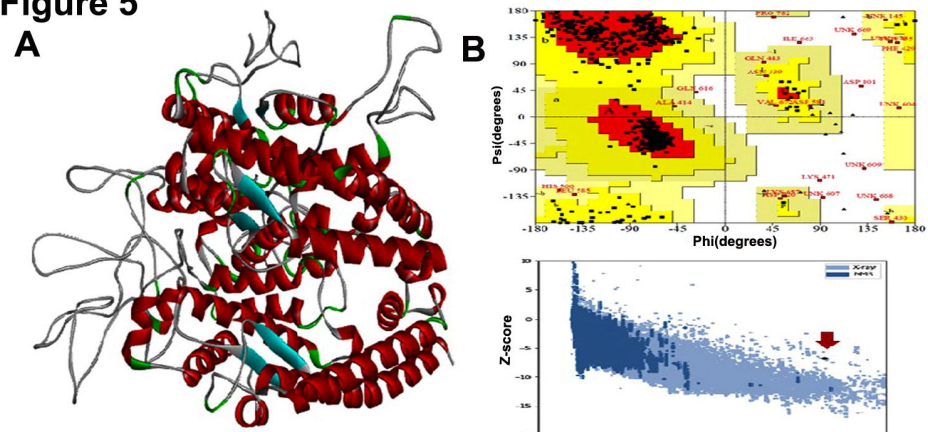

C

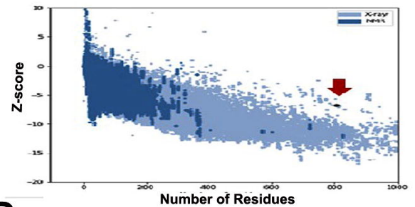

D

Number of Residues

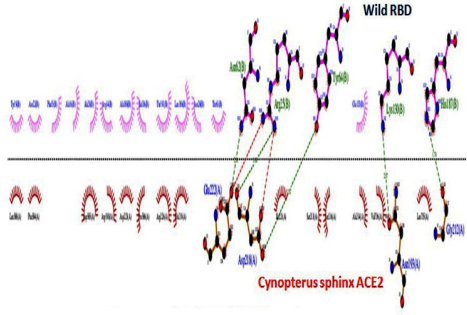

C. sphinx ACE2-Wild RBD complex

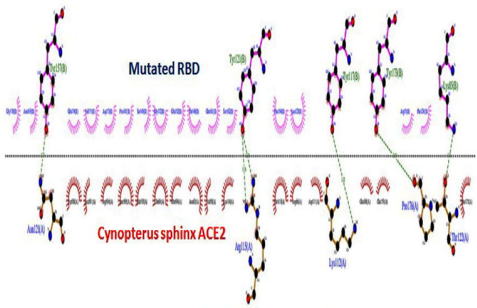

C. sphinx ACE2-Mutated RBD complex 

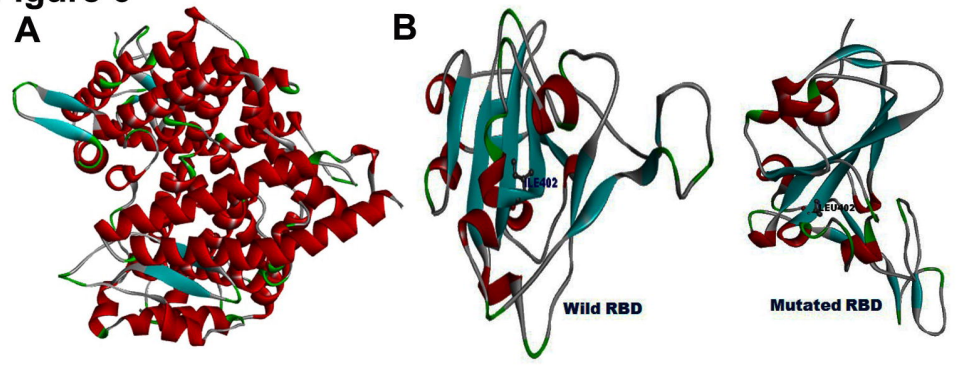

C

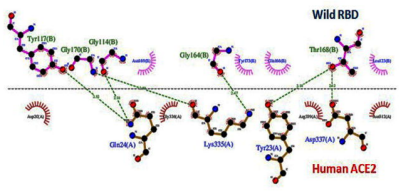

$$
\text { (1) }
$$$$
\text { Mutated RBD }
$$

H. sapiens ACE2-Wild RBD complex

H. sapiens ACE2-Mutated RBD complex 
Table 1: Patient information and sequence identity analysis of all the RMRC SARS-CoV-2 isolates

\begin{tabular}{|c|c|c|c|}
\hline Sl. No. & Sample ID & $\begin{array}{l}\text { RMRC Spike nucleotide } \\
\text { seq. identity with } \\
\text { reference }(\%)\end{array}$ & $\begin{array}{l}\text { RMRC } \\
\text { RBD } \\
\text { nucleotide } \\
\text { seq. } \\
\text { identity } \\
\text { with } \\
\text { reference } \\
(\%)\end{array}$ \\
\hline 1 & RMRC 2 & 99 & 100 \\
\hline 2 & RMRC 5 & 99 & 100 \\
\hline 3 & RMRC 6 & 99 & 100 \\
\hline 4 & RMRC 7 & 99 & 100 \\
\hline 5 & RMRC 22 & 99 & $99(\mathbf{M})$ \\
\hline 6 & RMRC 23 & 99 & 100 \\
\hline 7 & RMRC 24 & 99 & 100 \\
\hline 8 & RMRC 27 & 99 & 100 \\
\hline 9 & RMRC 28 & 99 & 100 \\
\hline 10 & RMRC 30 & 99 & 100 \\
\hline 11 & RMRC 38 & 99 & 100 \\
\hline 12 & RMRC 46 & 99 & 100 \\
\hline 13 & RMRC 90 & 99 & 100 \\
\hline 14 & RMRC 103 & 99 & 100 \\
\hline 15 & RMRC 104 & 100 & 100 \\
\hline 16 & RMRC 106 & 99 & 100 \\
\hline 17 & RMRC 108 & 99 & 100 \\
\hline 18 & RMRC 110 & 99 & 100 \\
\hline 19 & RMRC 112 & 99 & 100 \\
\hline 20 & RMRC 154 & 99 & 100 \\
\hline 21 & RMRC 155 & 99 & 100 \\
\hline 22 & RMRC 156 & 99 & 100 \\
\hline 23 & RMRC 157 & 100 & 100 \\
\hline 24 & RMRC 158 & 100 & 100 \\
\hline 25 & RMRC 159 & 99 & 100 \\
\hline 26 & RMRC 160 & 99 & 100 \\
\hline 27 & RMRC 162 & 99 & 100 \\
\hline 28 & RMRC 163 & 99 & 100 \\
\hline 29 & RMRC 164 & 99 & 100 \\
\hline 30 & RMRC 165 & 99 & 100 \\
\hline 31 & RMRC 166 & 99 & 100 \\
\hline 32 & RMRC 167 & 99 & 100 \\
\hline 33 & RMRC 168 & 99 & 100 \\
\hline 34 & RMRC 170 & 99 & 100 \\
\hline 35 & RMRC 171 & 99 & 100 \\
\hline
\end{tabular}


Table 2: Protein-protein interaction analysis of ACE2 and RBD (wild and Mutated) obtained by HawkDock web server

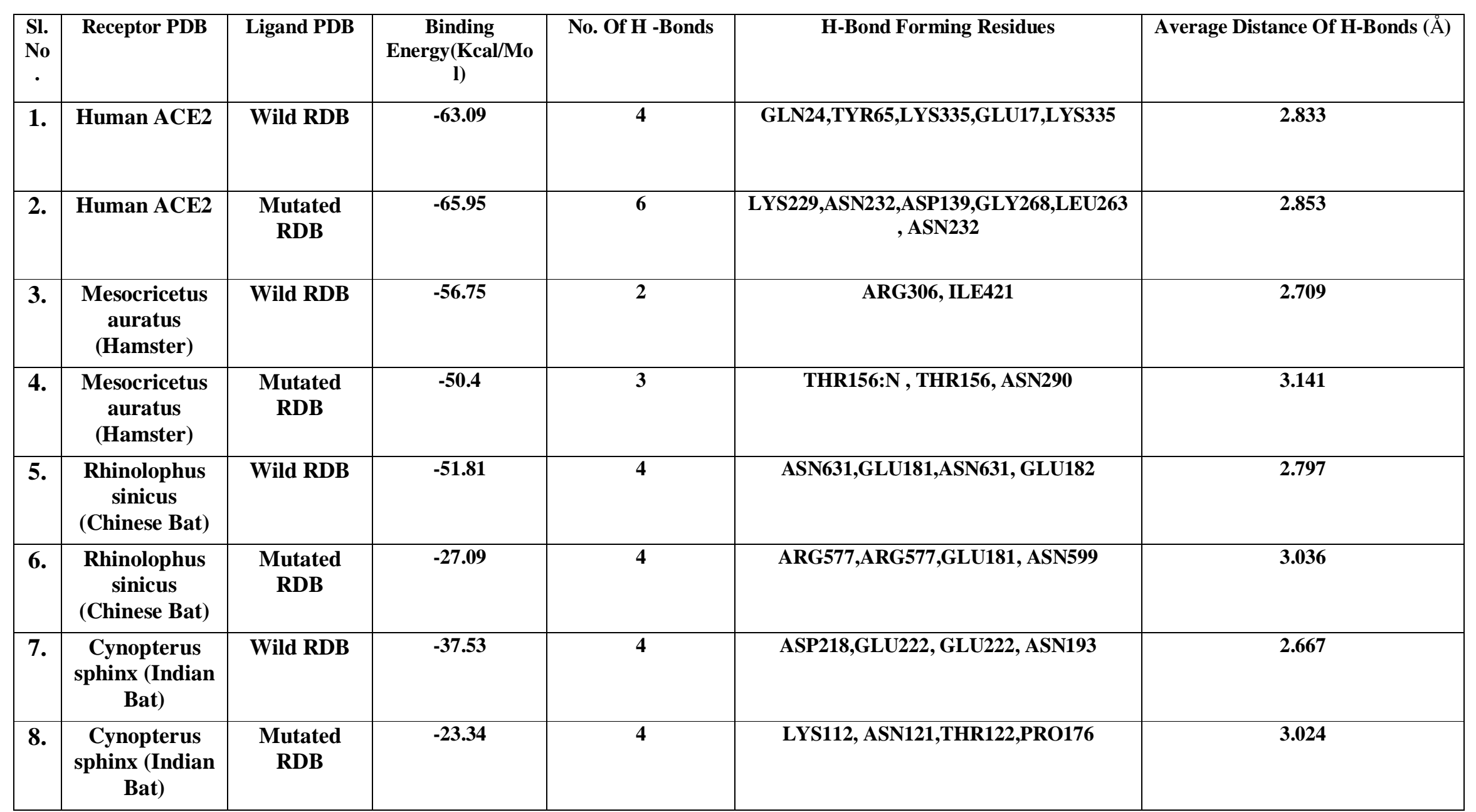




\begin{tabular}{|c|c|c|c|c|c|c|}
\hline 9. & $\begin{array}{c}\text { Manis } \\
\text { javanica } \\
\text { (Pangolin) }\end{array}$ & Wild RDB & -70.92 & 13 & $\begin{array}{c}\text { A:GLN86,LYS94,ASN213,ASN216,SER218 } \\
\text {,THR576,ARG192,ASN213,GLY214,GLU2 } \\
\text { 10,GLN598,GLU197,ILE744 }\end{array}$ & 2.807 \\
\hline $\begin{array}{c}10 \\
\cdot\end{array}$ & $\begin{array}{c}\text { Manis } \\
\text { javanica } \\
\text { (Pangolin) }\end{array}$ & $\begin{array}{c}\text { Mutated } \\
\text { RDB }\end{array}$ & -72.74 & 11 & $\begin{array}{c}\text { GLN86,LYS94, ASN213, ASN216,THR576, } \\
\text { ARG192, GLY214, GLU210,GLN598, } \\
\text { GLU197,ILE744 }\end{array}$ & 2.860 \\
\hline
\end{tabular}

